\title{
Autopilot Design for Unmanned Surface Vehicle based on CNN and $\mathrm{ACO}$
}

\author{
D. Zhao, T. Yang, W. Ou, H. Zhou
}

\author{
Dongming Zhao, Tiantian Yang, Wen Ou, Hao Zhou* \\ School of Automation \\ Wuhan University of Technology \\ Wuhan 430070, Hubei, China \\ dmzhao@whut.edu.cn, ttyang@whut.edu.cn,wenou@mail.hust.edu.cn \\ *Corresponding author; 18601157377@163.com
}

\begin{abstract}
There is a growing concern to design intelligent controllers for autopiloting unmanned surface vehicles as solution for many naval and civilian requirements. Traditional autopilot's performance declines due to the uncertainties in hydrodynamics as a result of harsh sailing conditions and sea states. This paper reports the design of a novel nonlinear model predictive controller (NMPC) based on convolutional neural network $(\mathrm{CNN})$ and ant colony optimizer $(\mathrm{ACO})$ which is superior to a linear proportional integral-derivative counterpart. This combination helps the control system to deal with model uncertainties with robustness. The results of simulation and experiment demonstrate the proposed method is more efficient and more capable to guide the vehicle through LOS waypoints particularly in the presence of large disturbances.

Keywords: USV, autopilot, predictive control, Convolution Neural Network (CNN), Ant Colony Optimization (ACO), rolling optimization.
\end{abstract}

\section{Introduction}

In recent years, The application of USVs is ever increasing in the fields of oceanography [13] [3], meteorology [7], military and commercial applications. Autopilot is a device for controlling the heading of USV in a truly autonomous mode, which plays a key role in course-keeping for USV [24]. When USV is working under harsh sea condition and high-risk environment, the performance of traditional autopilot declines in the presence of time-varying ocean disturbances, and measurement noises. A lot of autopilot designs for USV have been proposed to solve this problem. Gao et al. proposed a fuzzy neural network controller based on chaos neural network forecast model for the USV in complex sea condition $[9,15,17]$. An optimizing sliding mode cascade control structure is proposed to determine the optimal sliding surface parameters for sliding mode control of underactuated USV systems [18]. Further, nonlinear controller of USV is designed by backstepping method $[1,10]$. The fuzzy control approach is also applied to the control of USV $[8,11,19]$. Li et al. proposed an adaptive radial basis function based on neural network controller for the nonlinear control of USV which contains modeling errors and unknown bounded environment disturbances [4]. However, it's still an open problem that how to develop effective method for robustness and high-precision steering of USV in extreme sea condition.

This paper reports a kind of autopilot based on convolutional neural network(CNN) and ant colony optimizer. A NMPC based on CNN is used to compensate this predicted disturbance and the ant colony algorithm is used to predict the disturbances. This approach helps the autopilot to deal with model uncertainties and so on. The simulation and experiment results verified the efficacy of the proposed method.

The rest paper is organized as follows: Section 2 states the specifications of the USV and the problem formulation. Section 3 elaborates the autopilot design based on CNN and ACO. Section 4 provides the simulation results and experiment results. Section 5 gives the conclusion of this paper. 


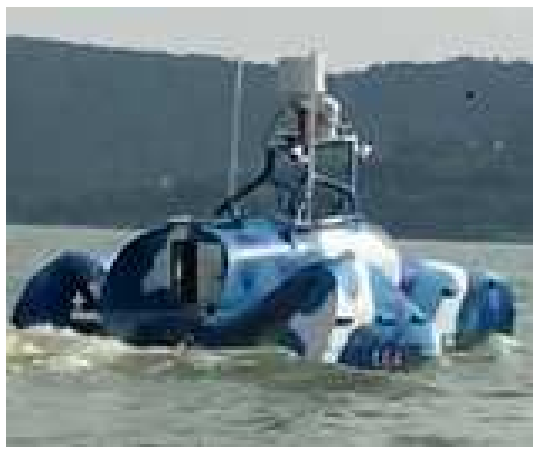

Figure 1: WH-01 USV

\section{Problem formulation}

Consider a robotic marine surface vehicle, called WH- 01 (as shown in Figure 1), whose specifications are listed in Table 1.The vehicle, designed in the control laboratory at WHUT(Wuhan University of Technology), has a hydraulic jet propeller and one rudder driven by servo motors for controlling its surge speed and heading. The maximum angular detection for rudder is $\pm 35^{\circ}$. It is equipped with navigation sensors including Beidou, 3-axis magnetometer, 3-axis accelerometer, and 3-axis rate gyro. The magnetometer and gyro are used to measure its yaw and yaw rate during trails, respectively. In addition, a $4 \mathrm{G}$ communication module is installed to control and monitor the vehicle from remote computer.

Table 1: SPECIFICATIONS OF WH-01 USV

\begin{tabular}{ll}
\hline Parameter & Value \\
\hline Length $/ m$ & 8.075 \\
Vertical length $/ m$ & 8 \\
draft depth $/ m$ & 0.6 \\
moulded depth $/ m$ & 1.15 \\
Full loaded displacement $/ T$ & 3.2 \\
Maximum speed $/ k n$ & 12 \\
Cruising speed $/ k n$ & 6 \\
Propulsion mode & water jet propulsion \\
\hline
\end{tabular}

Because of the interference of wind, waves and currents, the steering model of USV is expressed as follows:

$$
\begin{gathered}
T_{1} T_{2} \ddot{\Psi}+\left(T_{1}+T_{2}\right) \dot{\Psi}+\Psi+a \Psi^{3}=K \delta+K T_{3} \dot{\delta}+f_{a}+f_{\omega}+f_{l} \\
T_{1} T_{2}=\left(m+\lambda_{22}\right)\left(I_{z}+\lambda_{66}\right) / C \\
T=T_{1}+T_{2}-T_{3}
\end{gathered}
$$

where $\Psi$ is the heading angle of the USV, $\delta$ is the rudder angle of the USV , $T$ is the turning lag index, $T_{1} T_{2} T_{3}$ is the second-order turning lag index, $K$ is the turning ability index, $m$ is the mass of the USV, $f_{a}$ is equivalent disturbance rudder angle of the wind, $f_{\omega}$ is equivalent disturbance rudder angle of the disturbances due to waves, $f_{l}$ is equivalent disturbance rudder angle of the disturbances due to currents. 


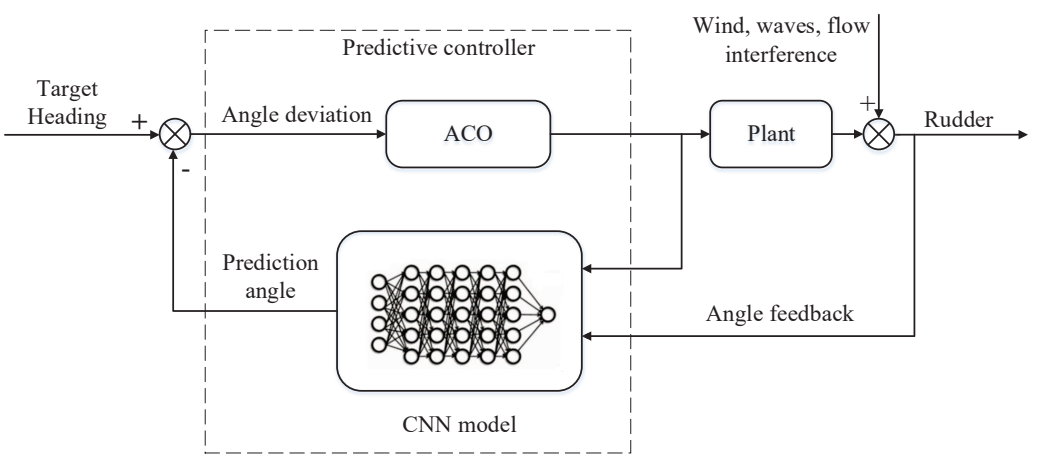

Figure 2: A block diagram of the autopilot based on CNN and ACO for USV

According to the calculation method in the literature [6] and the Zigzag experimental data of $\Psi / \delta=15^{\circ} / 15^{\circ}$ for the draft state in the Taihu Lake, it can be obtained that:

$$
K=2.9097, T=55.8855
$$

Zigzag experimental result is shown in table 2.

Table 2: ZIGZAG EXPERIMENTAL DATA OF USV

\begin{tabular}{|c|c|c|c|}
\hline$\overline{\text { Time } / s}$ & Rudder angle /o & Heading angle /o & Remark \\
\hline $1^{\prime} 82$ & 15 & 15 & - \\
\hline $5^{\prime} 82$ & 15 & 28.2 & $\begin{array}{c}\text { Maximum of } \\
\text { forward heading angle }\end{array}$ \\
\hline $9^{\prime} 44$ & -15 & 0 & - \\
\hline $12^{\prime} 68$ & -15 & -15 & $\begin{array}{c}\text { Maximum of } \\
\text { backward heading angle }\end{array}$ \\
\hline $15^{\prime} 62$ & 15 & -24.7 & - \\
\hline $23^{\prime} 06$ & 15 & 15 & $\begin{array}{l}\text { Maximum of } \\
\text { forward heading angle }\end{array}$ \\
\hline $26^{\prime} 56$ & -15 & 28.2 & - \\
\hline
\end{tabular}

\section{Robust adaptive autopilot}

The autopilot design of USV is based on convolutional neural network(CNN) and ant colony optimizer. Closed-loop state prediction and rolling horizon optimization are included in the predictive controller. Particularly, The future dynamic trend is predicted by the NMPC based on historical data of input and output. An ant colony algorithm acting as nonlinear optimizer is used to improve the rolling horizon. The predictive value is corrected by negative feedback and then compared with reference input. The difference between the predicted value and the expected value is minimized in the next period of time. The proposed autopilot design is shown in Figure 2.

\subsection{CNN model}

The architecture of CNN based model is shown in Figure 3. The delay lines with taps are represented by TDL(Tapped Delay Line). The CNN is in parallel with the process of system. 


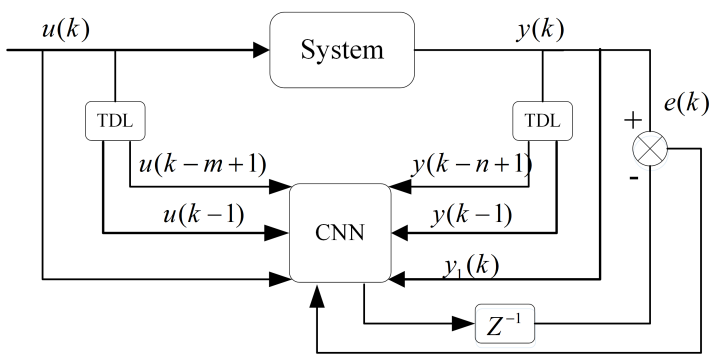

Figure 3: Architecture of CNN based model

The predictive error is used as training signal of the network. The single-step CNN's prediction model is defined as:

$$
y_{m}(k+1)=\hat{f}[y(k), y(k-1), \cdots, y(k-n+1) ; u(k), u(k-1), \cdots, u(k-m+1)]
$$

where $y(k)$ is the output of the heading angle at sampling time $k, u(k)$ is the input of the heading angle at sampling time $k, m$ is the system input order, $n$ is the system output order, $\hat{f}(\cdot)$ is the input and output mapping function of the CNN model, $y_{m}(k+1)$ is the predictive value of the heading angle.

Because the single-step neural network model has less predictive information, the multistep prediction method is used to improve the anti-interference and robustness of the autopilot. The prediction model of single-step neural network is used to infer the multi-step one, which is expressed as:

$$
y_{m}(k+p)=\hat{f}[y(k+p-1), \cdots, y(k+p-n) ; u(k+p-1), \cdots, u(k+p-m)]
$$

At sampling time $k, y(k+p-1), \cdots, y(k+1)$ is the actual output data of future, which can't be measured. So, the predictive value is used to replace the output of future. In addition, the predicted data before the $k t h$ moment can be replaced by historical data. So, the $p$ step predictive model is formulated as:

$$
y_{m}(k+p)=\hat{f}\left[y_{m}(k+p-1), \cdots, y_{m}(k+p-n) ; u(k+p-1), \cdots, u(k+p-m)\right]
$$

The CNN model structure of multi-step prediction is shown in Figure 4.

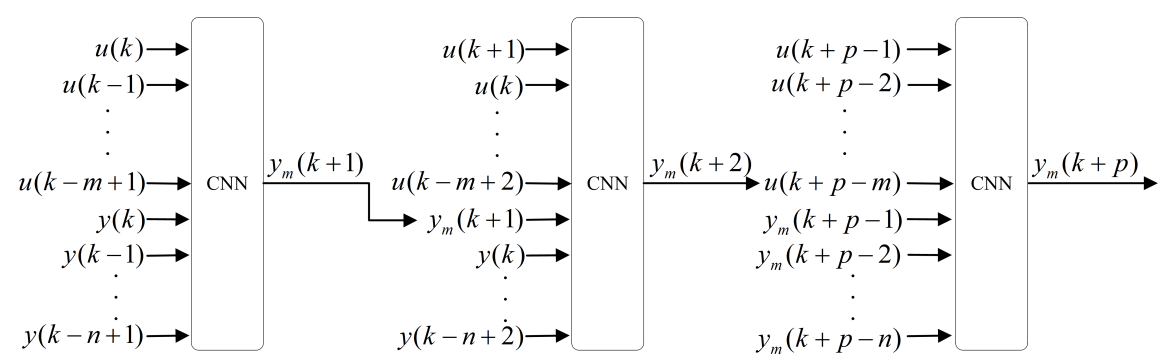

Figure 4: Structure of multi-step neural network prediction

\subsection{Feedback correction}

The reference trajectory function of the autopilot is based on the reference trajectory provided by the model control algorithm, which is expressed as:

$$
y_{\gamma}(k+1)=a y(k)+(1-a) \omega
$$


Because the predictive controller has the model mismatch problem, a large deviation is produced between prediction model and actual output of the object. The predictive output should be corrected to reduce the predictive error.

At sampling time $k$, we must calculate the error between the actual output value $y(k)$ and the output value predicted by the $\operatorname{model}\left(y_{m}(k)\right)$ at first. Then, the controller adds the error to the model predictive output $\left(y_{m}(k+1)\right)$ to get the closed-loop predictive output. The future closed-loop predictive output of $p$ step is as follows:

$$
y_{p j}(k+j)=y_{m}(k+j)+r_{j} e_{j}(k)+\beta_{j}\left[e_{j}(k)-e_{j}(k-1)\right]
$$

where $r_{j}$ is the error correction coefficient, $\beta_{j}$ is the change rate of correction coefficient, $e_{j}(k)=$ $y(k)-y_{m}(k)$ is the difference between actual output and the expected output, $j=1,2, \cdots, p$ is the number of predictive steps, $y_{p j}(k+j)$ is the predictive value of the output component after the $(k+j)$ moment, $y_{p j}(k+j)$ is the model predictor of the output component at sampling time $(k+j)$.

\subsection{Rolling optimization}

Usually, the difference between the predictive value of heading and the expected value of heading is supposed to be zero. The range of the control increment should not be too large. For this reason, the optimal law of predictive controller is determined by the performance index. The secondary performance indicator is used as the objective function. The optimal control objective function is introduced by:

$$
\begin{aligned}
J= & \frac{1}{2}\left\{\sum_{j=1}^{s} \sum_{i=1}^{P} q_{i j}\left[y_{p j}(k+i)-y_{\gamma j}(k+j)\right]^{2}\right. \\
& \left.+\sum_{j=1}^{\gamma} \sum_{i=1}^{M} \lambda_{i j}\left[u_{j}(k+i-1)-u_{j}(k+i-2)\right]^{2}\right\}
\end{aligned}
$$

where $P$ is the length of predictive time domain, $M$ is the length of time domain, $M \leq P$, $q_{j}$ is the error weighting factor, $\lambda_{j}$ is the control incremental weighting factor, $y_{j}(k+j)$ is the reference trajectory of the output at $k+j$ moment.

The ant colony optimizer( $\mathrm{ACO}$ ) is used to maximize the objective function of the controller within the range of rudder angle, which has good robustness and can be implemented in parallel. The implementation steps of the ACO are enumerated below:

1. Initialize the ant pheromone distribution, set the number of iterations;

2. 10 ants are initialized in the neighborhood, moving according to the following transfer probability. For each ant $i$, the objective function $J_{i}$ is defined, the transfer probability for ant $i$ at time $k$ is as follows:

$$
p_{i j}(k)=\frac{\left[\tau_{j}(k)\right]^{1.2}\left[\triangle J_{i j}(k)\right]^{2}}{\sum_{r}\left[\tau_{r}(k)\right]^{1.2}\left[\triangle J_{i r}(k)\right]^{2}}
$$

where $\tau_{j}(k)$ is the j-neighborhood attracting intensity of the ants at $k$ time. $\triangle J_{i j}=J_{i}-J_{j}$ is the difference value of the target function.

3. Calculate the objective function value of each ant, and record the optimal control sequence in the current ant colony; 
4. Revise the intensity of pheromone according to the pheromone update equation, following the next equation:

$$
\left\{\begin{array}{c}
\tau_{j}(k+1)=0.7 \tau_{j}(k)+\sum \triangle \tau_{j} \\
\triangle \tau_{j}=1 / J_{j}
\end{array}\right.
$$

5. Number of iterations $N=N+1$

6. If the number of iterations does not reach the ending iteration number 100, the second step is returned, otherwise, the loop is terminated and the optimal control sequence is outputted.

\section{Results and Discussion}

\subsection{Simulation results}

The disturbance of wind and wave on USV is simulated using white noise and a second-order wave transfer function [26]. The disturbance of ocean current is treated as a constant value.

$$
y(s)=K_{c} \omega(s) \frac{K_{\omega} s}{s^{2}+2 \zeta \omega_{0} s+\omega_{0}^{2}}
$$

where $\omega(s)$ is a Gaussian white noise with a mean of zero, the value of power spectrum density is 0.1 , and $K_{c}=5$ is a constant disturbance coefficient, $\zeta=0.3$ is a damp coefficient, $K_{\omega}=0.42$ is the gain constant, $\omega_{0}=0.606$ is the frequency of the dominant wave.

In the case of wind, wave and current interference, when the setting heading angle is $15^{\circ}$, the effect of traditional controller and predictive controller simulated using matlab is shown in the following figure.

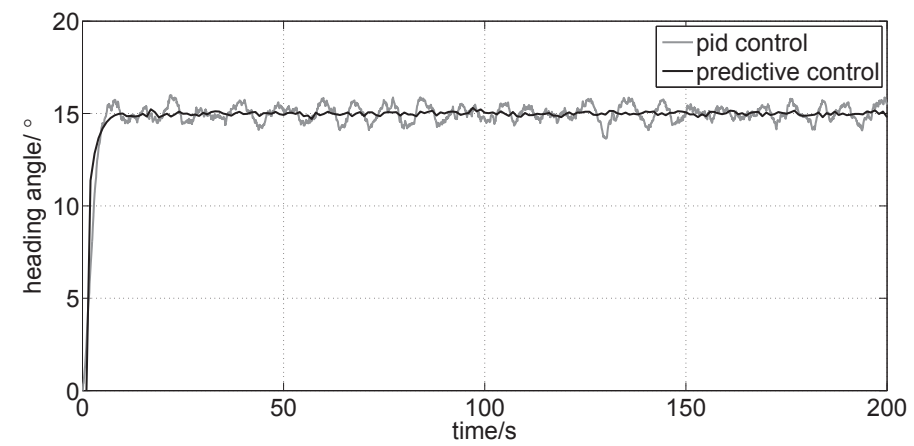

Figure 5: Heading using PID(Proportion Integration Differentiation) and the MPC

It is shown from Fig.5, Fig.6 that the predictive controller based on CNN has good control accuracy, robustness and fault tolerance. Compared with the traditional model predictive control, this controller's stability has become stronger with external disturbances, which can realize the stable path tracking with the characteristics of strong anti-interference. Finally, the problem of model mismatch in conventional generalized predictive control is also solved by this proposed method. 


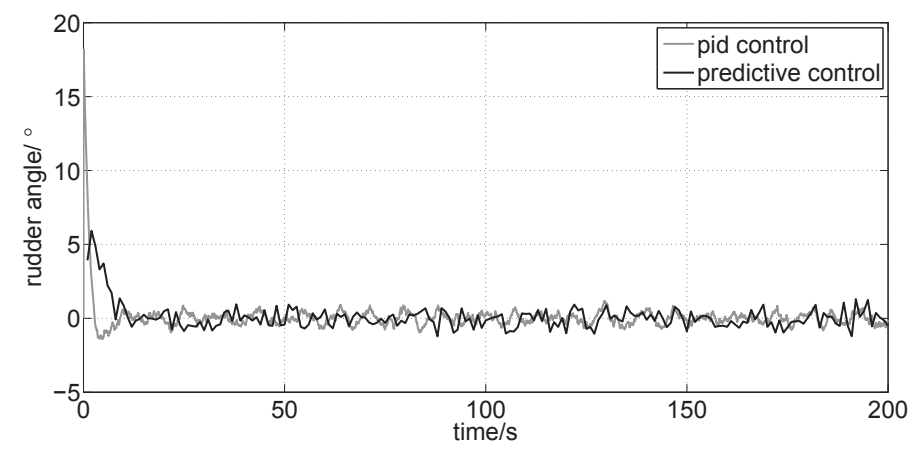

Figure 6: Rudder angle using PID and the MPC

Table 3: Latitude and longitude of the target point

\begin{tabular}{ccc}
\hline \multirow{2}{*}{ target number } & \multicolumn{2}{c}{ Coordinate } \\
\cline { 2 - 3 } & longitude & latitude \\
\hline 1 & $120^{\circ} 08^{\prime} 03.7^{\prime \prime} E$ & $31^{\circ} 26^{\prime} 31.4^{\prime \prime} N$ \\
2 & $120^{\circ} 08^{\prime} 07.7^{\prime \prime} E$ & $31^{\circ} 26^{\prime} 27.9^{\prime \prime} N$ \\
3 & $120^{\circ} 08^{\prime} 06.5^{\prime \prime} E$ & $31^{\circ} 26^{\prime} 23.1^{\prime \prime} N$ \\
4 & $120^{\circ} 08^{\prime} 02.3^{\prime \prime} E$ & $31^{\circ} 26^{\prime} 21.0^{\prime \prime} N$ \\
5 & $120^{\circ} 07^{\prime} 57.4^{\prime \prime} E$ & $31^{\circ} 26^{\prime} 24.3^{\prime \prime} N$ \\
6 & $120^{\circ} 07^{\prime} 59.0^{\prime \prime} E$ & $31^{\circ} 26^{\prime} 29.4^{q \prime \prime} N$ \\
\hline
\end{tabular}

\subsection{Experimental results}

The predictive controller is applied to the WH-01, in which the course control experiment was carried out in Taihu Lake, Wuxi, Jiangsu province. Six target points are selected for the typical trajectory experiment in the lake, of which the latitude and longitude are shown in Table 2. The USV traveled at the average speed of 12 knot during the experiment. This simulation data is derived from the average of three experimental data.

Fig. 7 and Fig. 8 show the waypoints follow by the USV by the predictive controller and the PID scheme. Fig.9 and Fig.10 illustrate the distance of the vehicle from next waypoint and total time taken to operate under the predictive controller and the PID scheme. Fig.11 and Fig.12 demonstrate mean average deviation of the waypoints by the predictive controller and the PID scheme. It can be shown from Fig.7, Fig.8 and Table 4 that the USV can also pass through the target points when disturbed by wind and waves. It is observed that the predictive controller is

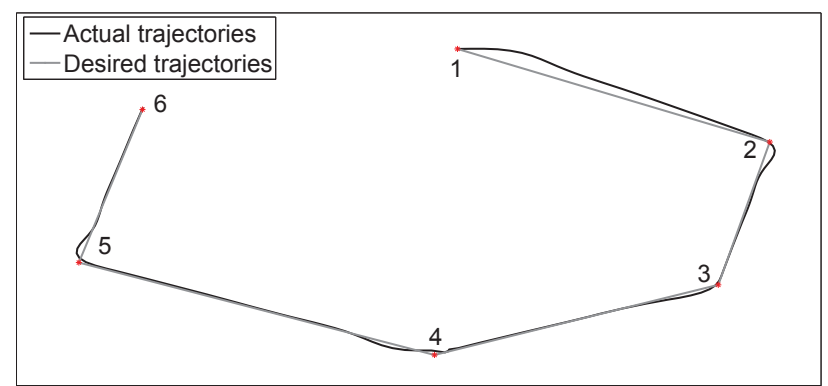

Figure 7: Waypoints followed by the Predictive controller systems 


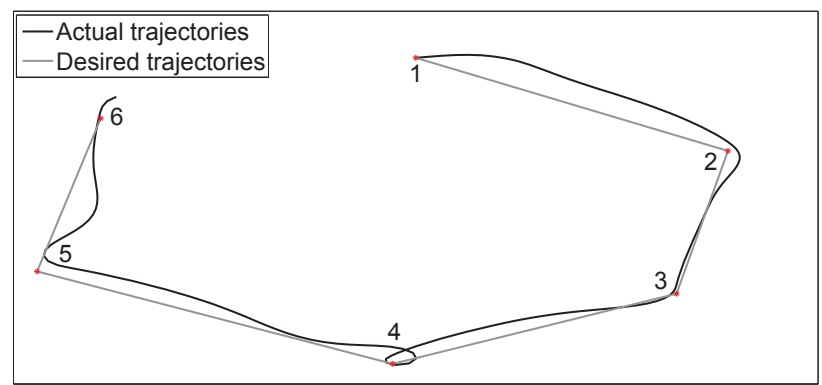

Figure 8: Waypoints followed by the PID GC systems

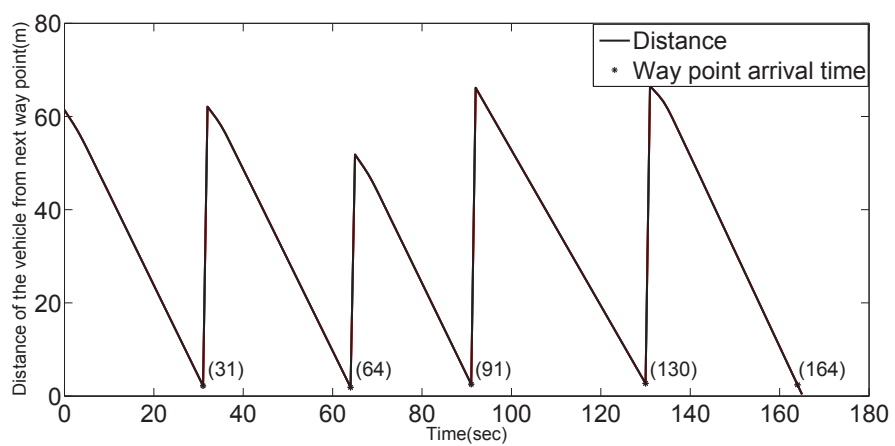

Figure 9: Distance of the vehicle from next waypoint and total time taken to operate by the Predictive controller systems

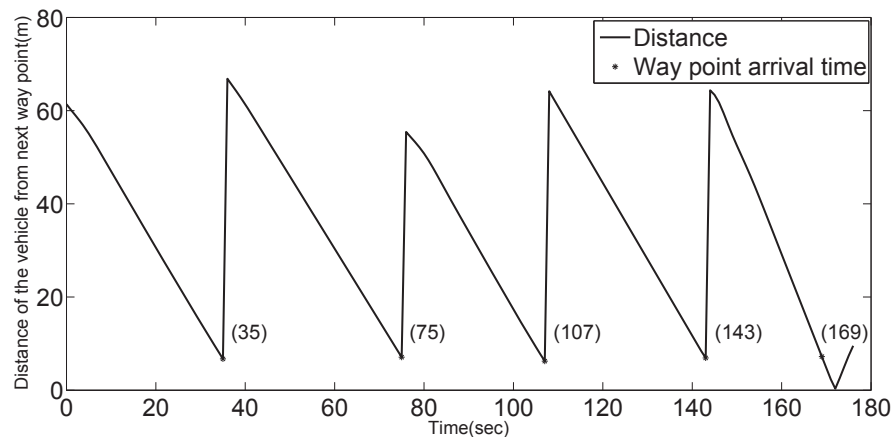

Figure 10: Distance of the vehicle from next waypoint and total time taken to operate by the PID GC systems(guidance and control systems)

Table 4: Average performance measures of predictive control

\begin{tabular}{cccc}
\hline Case & $\begin{array}{c}\text { Number of } \\
\text { target points arriving }\end{array}$ & $\begin{array}{c}\text { Total } \\
\text { distance }(\mathrm{m})\end{array}$ & $\begin{array}{c}\overline{r d} \\
(\mathrm{~m} / \mathrm{s})\end{array}$ \\
\hline $\operatorname{PID}\left(K_{p}=8, T_{i}=8, T_{d}=0\right)$ & 6 & 393.57 & 8.01 \\
Predictive controller & 6 & 347.98 & 4.23
\end{tabular}




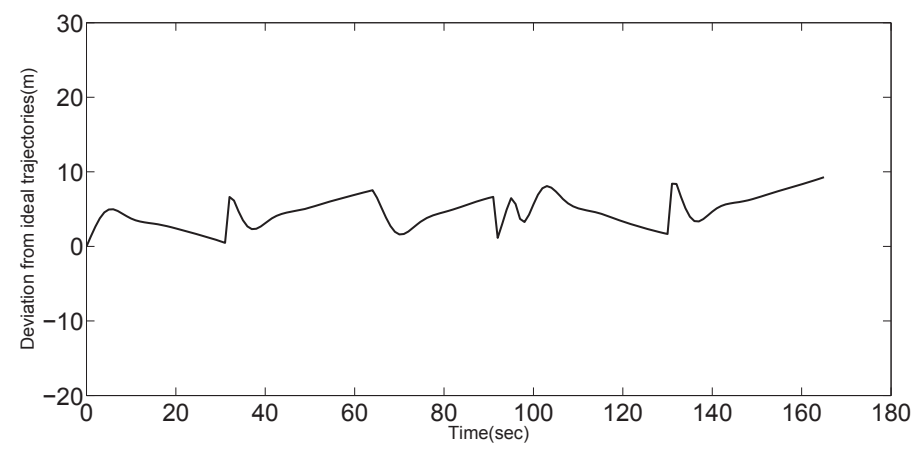

Figure 11: Mean average deviation of the waypoints by the Predictive controller systems

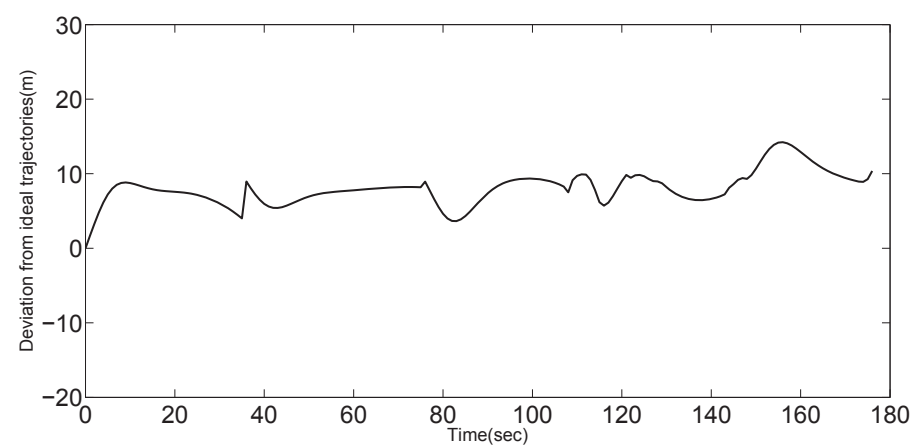

Figure 12: Mean average deviation of the waypoints by the PID GC systems

more efficient and capable to control the USV tracking the target points especially in the case of disturbances.

\section{Conclusion}

A predictive controller based on convolutional neural network $(\mathrm{CNN})$ is designed for autopiloting an USV. It is shown from the experimental results that the predictive controller has good robustness and anti-jamming. In extreme sea condition, the problem of control overshooting and poor anti-interference is solved by the algorithm. In addition, the difficulty in hydrodynamic modeling can be solved by the proposed approach. Finally, the feasibility of the method is verified by numerical simulations and experiments on lake.

However, the steering mathematical model based on the CNN needs to be improved in the future. Particularly, the training samples accumulated from the model experiment, the actual ship test and expert knowledge need to be analyzed. In these conditions, it is difficult to build the nonlinear steering law, which is worthy of further research.

The next work will be related to research the optimal strategy and design a new predictive controller with good accuracy, adaptability and robustness. The influence of the designed parameters on the stability and other control performance can be analyzed in the system. The robustness of the predictive control system can be researched when there has modeling error and disturbance. Then, the different optimization strategies can be used to derive the different controller structure. Therefore, a new rolling optimization strategy needs to be researched. 


\section{Acknowledgement}

The work was supported by National High-Tech Research and Development Program 2015AA015904.

\section{Bibliography}

[1] Abdolmalaki, R.Y.; Mahjoob, M.J.; Abbasi,E. (2013); Fuzzy LQR Controller for Heading Control of an Unmanned Surface Vessel, International Workshops in Electrical-Electronics Engineering, 2013.

[2] Annamalai, Andy S.K. (2014); An adaptive autopilot design for an uninhabited surface vehicle, PhD thesis, University of Plymouth, 2014.

[3] Brando, V.; Lovell, J.; King, E.; Boadle, D.; Scott, R.; Schroeder, T. (2016); The potential of autonomous ship-borne hyperspectral radiometers for the validation of ocean color radiometry data, Remote Sensing, 8(2), 150, 2016.

[4] Dou, C.X. (2003); Design of Fuzzy Neural Network Controller Based on Chaos Neural Network Forecast Model and Application, Systems Engineering-theory \& Practice, 23(8), 48-52, 2003.

[5] Dzitac, I. (2015); Impact of Membrane Computing and P Systems in ISI WoS. Celebrating the 65th Birthday of Gheorghe Păun, International Journal of Computers Communications \& Control, 10(5), 617-626, 2015.

[6] Fan, S.Y. (1988); Ship maneuverability, National Defense Industry Press, 1988.

[7] Fitzpatrick, P.J.; Lau, Y.;Moorhead, R.; Skarke, A.; Merritt, D.; Kreider, K.; Brown, C.; Carlon, R.; Hine, G.; Lampoudi, T.; Leonardi, A.P. (2007); A review of the 2014 Gulf of Mexico Wave Glider field program, Marine Technology Society Journal, 49(3), 64-71, 2007.

[8] Gao, F. (2012); Design and Research of Key Technologies for a New AUV in Complex Sea Conditions, PhD thesis, National University of Defense Technology, 2012.

[9] Jiang, L.; Mu, D.; Fan, Y.; Wang, G.; Zhao, Y. (2016); Study on USV model Identification and nonlinear course control, Computer Measurement \& Control, 24, 133-136, 2016.

[10] Larrazabal, J.M.; Penas, M.S. (2016); Intelligent rudder control of an unmanned surface vessel, Expert Systems with Applications, 55, 106-117, 2016.

[11] Li, C.; Zhao, Y.; Wang, G. (2016); Adaptive RBF neural network control for USVcourse tracking, International Conference on Information Science \& Technology, 285-290, 2016.

[12] Li, R. (2012); Research and application on Generalized predictive control based on particle swarm optimization algorithm, MSc thesis, LanZhou JiaoTong University, 2012.

[13] Liu, C.; Chu, X.; Wu, Q.; Wang, G. (2014); USV development status and prospects, China Shipbuilding, 194-205, 2014.

[14] Mcninch, L.C.; Muske, K.R.; Ashrafiuon, H. (2008); Model-based predictive control of an unmanned surface vessel, IASTED International Conference on Intelligent Systems and Control, 385-390, 2008. 
[15] Mcninch, L.C.; Ashrafiuon, H. (2011); Predictive and sliding mode cascade control for Unmanned Surface Vessels, American Control Conference, 145(2), 184-189, 2011.

[16] Moe, S.; Pettersen, K.Y. (2016); Set-based Line-of-Sight (LOS) path following with collision avoidance for underactuated unmanned surface vessel, In 24th Mediterranean Conference Control and Automation, 402-409, 2016.

[17] Mu, D.; Zhao, Y.; Wang, G.; Fan, Y.; Bai, Y. (2016); USV model identification and course control, Sixth International Conference on Information Science and Technology, 263-267, 2016 .

[18] Mu, D.; Zhao, Y.; Wang, G.; Fan, Y.; Bai, Y. (2016); Course control of USV based on fuzzy adaptive guide control, Control and Decision Conference, 6433-6437, 2016.

[19] Ni, H. (2006); Research on Predictive Control Method Based on Neural Network and Its Application, MSc thesis, Central South University, 2006.

[20] Pan, L.; He, C.; Tian, Y.; Su, Y.; Zhang, X. (2017); A region division based diversity maintaining approach for many-objective optimization, Integrated Computer-Aided Engineering, 24(3), 1-18, 2017.

[21] Pan, L.; Păun, G. (2009); Spiking neural P systems with anti-spikes, International Journal of Computers Communications \& Control, 4(3), 273-282, 2009.

[22] Păun, G. (2016); Membrane Computing and Economics: A General View, International Journal of Computers Communications \& Control, 11(1), 105-112, 2016.

[23] Shen, Y.; Cheng, Y.; Ji, Z. (2006); Controller Design for Asynchronism Motor Based on Multi-step Predictive Neural Network, Small \& Special Electrical Machines, 34(12), 34-36, 2006.

[24] Sonnenburg, C.; Woolsey, C.A. (2012); An experimental comparison of two USV trajectory tracking control laws, Oceans, 1-10, 2012.

[25] Wang, C.S.; Xiao, H.R.; Han, Y.Z. (2013); Applications of ADRC in Unmanned Surface Vessel Course Tracking, Applied Mechanics \& Materials, 427-429:897-900, 2013.

[26] Wang, Y.D. (2014); Based on auto disturbance rejection control algorithm for course autopilot of unmanned surface vessel design, MSc thesis, Dalian Maritime University, 2014.

[27] Wu, G.; Jin, Z.; Lei, W.; Qin, Z. (2009). Design of the Intelligence Motion Control System for the High-Speed USV, Intelligent Computation Technology and Automation, 3, 50-53, 2009 .

[28] Yang, J.F. (2007); Ant colony algorithm and its application research, PhD thesis, Zhejiang University, 2007.

[29] Yang, L. (2013); Analysis and Design of Simplified Dual Neural Network Based Model Predictive Controller, MSc thesis, Shanghai Jiao Tong University, 2013. 